\title{
Otium et écriture dans Un flâneur en Patagonie de William Henry Hudson (1893)
}

Otium and Writing in William Henry Hudson's Idle Days in Patagonia (1893)

Heidi Liedke

Traducteur : Anna Pevoski et Clara Schwarze

\section{(2) OpenEdition}

\section{Journals}

Édition électronique

URL : https://journals.openedition.org/recherchestravaux/815

DOI : 10.4000/recherchestravaux.815

ISSN : 1969-6434

Éditeur

UGA Éditions/Université Grenoble Alpes

\section{Édition imprimée}

Date de publication : 1 juin 2016

Pagination : 107-127

ISBN : 978-2-84310-325-4

ISSN : 0151-1874

\section{Référence électronique}

Heidi Liedke, « Otium et écriture dans Un flâneur en Patagonie de William Henry Hudson (1893)»,

Recherches \& Travaux [En ligne], 88 | 2016, mis en ligne le 01 janvier 2017, consulté le 29 octobre 2021.

URL : http://journals.openedition.org/recherchestravaux/815; DOI : https://doi.org/10.4000/

recherchestravaux.815 
Heidi LIEDKE

Université Albert-Ludwig de Fribourg-en-Brisgau, SFB ıoI5 «Muße»

\section{Otium $^{1}$ et écriture dans Un flâneur en Patagonie de William Henry Hudson (1893)}

(Traduit de l'anglais par Anna Pevoski et Clara Schwarze)

Marcher et regarder autour de soi, s'arrêter, revenir sur ses pas, observer - ces activités, en rapport avec «son enthousiasme pour ses sujets ${ }^{2} »-$ peu importe de quelle obscurité, banalité apparente ou étrangeté - peuvent servir de mots clefs pour décrire William Henry Hudson et son écriture. Dans son essai «W. H. Hudson - Poet Strayed into Science», Ezra Pound ajoute un aspect supplémentaire qui ne doit pas être omis lorsqu'on évoque Hudson, à savoir le conflit, perçu par beaucoup de ses critiques, entre sa science et son entreprise littéraire «romantique» (c'est-à-dire relative aux affaires du cœur), et il mentionne aussi un autre exemple pour illustrer ce qui est désigné ici comme étant un sujet apparemment banal : la description que fait Hudson dans un chapitre de The Shepherd's Life d'un chat devant la gare d'un village anglais. Toujours selon Pound, on se surprend à être envoûté par ce chapitre, ce qui implique que l'écriture d'Hudson est de «l'art d'une grande qualités " ", une idée qui sera reprise ci-dessous.

Né à Quilmes en Argentine en I84I de parents originaires de NouvelleAngleterre ${ }^{4}$, Hudson n'émigra en Angleterre qu'en I874, après leur décès 5 .

I. J'utilise dans cet article le terme "oisiveté» (idleness) pour faire référence à l'otium.

2. E. Pound, «Hudson: Poet Strayed into Science», The Little Review. W. H. Hudson Number, $\mathrm{n}^{\circ}$ 7, I, I967 [I920-I92I], p. I3-I7, p. I3.

3. Ibid.

4. Selon F. Madox Ford, après la mort d'Hudson «son biographe assidu et dévoué, Mr. Morley Roberts, a fait des recherches pour découvrir finalement que le père d'Hudson était né dans l'État du Maine vers I8I4, son grand-père paternel ayant quitté l'Ouest de l'Angleterre pour le Maine peu avant la Déclaration d'Indépendance. Du côté maternel, en revanche, ses ancêtres ont vécu aux États-Unis pendant très longtemps» (F. Madox Ford, "W. H. Hudson", The American Mercury, 1936, p. 306-317, p. 309).

5. En Amérique du Sud, il est connu sous le nom de Guillermo Enrique Hudson. Il existe plusieurs biographies sur lui en espagnol, par exemple : E. Martínez Estrada, El Mundo maravilloso de Guillermo Enrique Hudson, Mexico, Fondo de Cultura Económica, I95I. 
En effet, pendant longtemps, ni ces simples dates ni son lieu de naissance ne furent certains car Hudson aima toujours garder le mystère sur sa vie et avait brûlé bon nombre de documents privés avant sa $\operatorname{mort}^{6}$. En soi, cela n'aurait pas été si atypique pour un homme de lettres. La volonté d'exercer, après la mort, un contrôle sur sa propre image est une préoccupation majeure de beaucoup d'écrivains, le plus célèbre exemple d'entre eux étant George Eliot. Mais il semblerait qu'Hudson ait été particulièrement déterminé à préserver sa vie privée et il a toujours, depuis son enfance, été jaloux de sa tranquillité. À cette époque, comme l'écrit Ruth Tomalin dans la biographie qu'elle lui consacre, sa mère le vit souvent «rêvassant, quelque part dans un endroit reculé, regardant, apparemment, dans le vide. À ce moment-là, elle réalisa qu'il était en train de regarder "un oiseau - un couple de petits gobe-mouches écarlates construisant un nid de lichen sur un pêcher, ou une telle chose merveilleuse" 7 ".

Dans cette contribution, je veux évoquer l'oisiveté selon la conception d'Hudson - d'après son journal de voyage en Patagonie durant l'été i87oI871, Un flâneur en Patagonie (Idle Days in Patagonia) - et l'importance de celle-ci pour son processus artistique. L'oisiveté se présente sous une forme hétérogène dans la pratique d'Hudson : initialement, celle-ci est de nature purement physique et extérieure lorsqu'il est obligé de garder le lit à cause d'une blessure, plus tard, elle est intériorisée et finit par faire partie de son personnage. Dans ses écrits, elle apparait principalement comme provoquée par le silence et la solitude. Dans ses souvenirs, elle prend la forme de crises et d'éclats étrangement poétiques que je nommerai «ralentisseurs». En ce qui concerne le Flâneur en Patagonie, une charmante sobriété est obtenue grâce à l'effet d'équilibre créé par les références répétées à des chapitres précédents ou suivants. Grâce à elles, Hudson donne à tout moment à ses lecteurs une idée exacte de l'endroit où ils se trouvent dans le texte. À cet égard les phrases donnent en effet l'impression d'être issues d'une thèse de doctorat ${ }^{8}$. Ce système de contrepoids, même s'il n'apparaît que dans la première moitié du livre, contraste avec sa matière principale : la tendance d'Hudson à flâner, à s'attarder, à divaguer et, surtout, à méditer. Il sera donc particulièrement intéressant de différencier les formes explicites et implicites de l'oisiveté - vécues ou textuelles - que l'on peut trouver dans l'œuvre.

6. Voir R. Tomalin, W. H. Hudson. A Biography, Londres, Faber and Faber, 1982, p. I5.

7. Ibid., p. 22.

8. En fin d'ouvrage sont indexés sur quatre pages les noms d'oiseaux (donnés aussi en latin), de lieux et d'auteurs mentionnés dans le texte. Cette pratique n'est pas nécessairement représentative d'un journal de voyage de cette époque. 
Il est remarquable qu'Hudson ait écrit Un flâneur en Patagonie en I893 alors qu'il vivait à la limite de l'extrême pauvreté avec sa femme Emily : sa motivation immédiate était donc d'écrire pour gagner de l'argent, comme s'il n'était qu'un gratte-papier. Il ne pouvait pas se permettre de passer de longues périodes à découvrir la campagne anglaise - il ne le fit que plusieurs années plus tard, avec pour résultat Nature in Downland (1900), Hampshire Days (1903) et The Shepherd's Life (I9IO) : son rôle de chroniqueur de la campagne anglaise s'en trouve confirmé. C'est ainsi que ses premières publications", The Naturalist in La Plata (I892) et Idle Days in Patagonia, reposent entièrement sur ses expériences en Amérique du Sud ${ }^{\mathrm{i}}$. Pour s'en sortir financièrement, il puisait alors dans des sources anciennes : c'est le cas pour Un Flâneur en Patagonie, c'est-à-dire une "série d'ébauches brillantes et suggestives», comme l'estime avec enthousiasme un article de la Saturday Review ${ }^{\text {II }}$. Au vu de sa mauvaise situation financière, on ne devrait pas sous-estimer l'importance de ce retour vers une période relativement insouciante et le fait qu'il pouvait en tirer quelque chose de fructueux. D'autre part, il était sans aucun doute un expert, non seulement des oiseaux, mais aussi de toutes les cultures et traditions des peuples d'Amérique du Sud. C'est aussi ce qui fit dire en 1946 à Herschel Brickell, alors responsable des relations culturelles de l'ambassade des États-Unis à Bogota :

Les avantages qu'offre la lecture d'Hudson pour découvrir l'Amérique du Sud sont [...] nombreux. Tout d'abord, il est un fin connaisseur de ces sujets. Ensuite, il est sans aucun doute un bon écrivain. Et enfin, même lorsque tous les sujets intéressants et importants à son époque seront dépassés, les thèmes dont il parle perdureront ${ }^{12}$.

Bien que le jugement de Brickell soit un peu disproportionné, il met l'accent sur trois aspects caractéristiques de l'écriture d'Hudson qui feront également partie de mon interprétation du Flâneur en Patagonie : il adapte son style au sujet, et sa narration est authentique; le texte varie cependant quant à son style

9. Hudson avait déjà écrit des travaux ornithologiques pour la Royal Zoological Society avant les années i870. Comme Shrubsall et Coustillas le relèvent, "on ne savait que très peu sur les oiseaux d'Argentine lorsqu'Hudson, âgé de 25 ans, fut envoyé par la Smithsonian Institution of Washington D. C. pour recueillir, dans un but scientifique, les plumages des oiseaux. Cela devait s'avérer un autre jalon dans son parcours littéraire parce qu'il entra ainsi en contact avec le directeur administratif de la Zoological Society of London, Dr Philip Lutley Sclater, qui éditera douze de ses lettres sur les oiseaux sud-américains dans la revue de la société" (D. Shrubsall et P. Coustillas [dir.], Landscapes and Literati. Unpublished Letters of W. H. Hudson and George Gissing, Silisbury, Russell, 1985, p. 2).

Io. Ibid., p. 3.

II. I893, p. 36I.

I2. H. Brickell, «W. H. Hudson - Bridge-Builder Between the Americas», Hispania, no 29 , I, I946, p. 98-IOI, p. IOO. 
et possède une qualité littéraire dont l'auteur profite pour examiner en détail le paysage de Patagonie en tant que vecteur d'oisiveté; enfin, ses réflexions oisives acquièrent une valeur abstraite transhistorique dans la mesure où elles évoquent des thèmes qui ne sont spécifiques ni au temps ni au lieu.

On est tout d'abord frappé par la diversité des thèmes auxquels s'intéresse Hudson, par ses choix inhabituels de sujets et par la variété de son style qui capte le lecteur. Sa capacité à changer de style à l'intérieur d'une même œuvre - ce qui est le cas aussi dans le Flâneur - est également impressionnante. Cela s'explique partiellement par le fait qu'il ne se sentait pas appartenir à une profession en particulier qui l'aurait obligé à écrire dans une veine spécifique. À l'occasion de sa disparition, le Times écrit qu'il était «sans égal en tant qu'écrivain anglais de la nature ${ }^{13} »$. Aussi fut-il assigné à une catégorie, alors même qu'il parlait de lui-même comme d'un «naturaliste ${ }^{\mathrm{I} 4} »$. Lors de sa nomination à la Royal Society of Literature's Academic Committee, il a même écrit que ce serait absurde de le qualifier d' "homme de lettres ${ }^{15}$ », et plus encore, pourraiton ajouter, d' "homme de lettres du canon anglophone», étant donné qu'il a d'abord écrit en espagnol.

Mais malgré sa réticence à être considéré comme un romancier, le début de son journal de voyage se lit comme un roman d'aventures dans lequel le lecteur plonge directement. Hudson et son équipage vont sur un bateau à vapeur vers le Rio Negro. Le moteur du navire "hal[ète] comme un cœur humain surmenér ${ }^{16}$, dans une atmosphère de danger imminent et de mort. L'incipit ressemble à des passages de Moby Dick d'Herman Melville (ce n'est pas l'unique parallèle que l'on discutera) et il est probable qu'Hudson s'est inspiré de son style. Tomalin compare l'incipit avec The Tempest de Shakespeare, c'est-à-dire «avec une tempête en haute mer, un navire qui coule, une voix criant "nous sommes perdus" », et il explique que «le mélange de voyage, d'aventure et de débat sur la théorie de l'évolution avait un fort attrait pour les gens de la fin de l'époque victorienne ${ }^{17} »$. L'atmosphère au début des souvenirs de voyage d'Hudson ne se poursuit cependant pas dans la suite du texte. Elle s'arrête même de manière plutôt abrupte après le premier chapitre, ce qui pourrait d'abord, d'un point de vue littéraire, sembler quelque peu inconséquent. On apprend qu'Hudson

13. "William Henry Hudson", The Times, I9 août 1922, p. I3.

I4. F. Madox Ford le cite s'exclamant avec enthousiasme: "Je ne suis pas l'un de vos maudits écrivains : je suis un naturaliste de la Plata.» («W. H. Hudson», art. cité, p. 309.)

15. Voir D. Shrubsall et P. Coustillas (dir.), Landscapes and Literati, ouvr. cité, p. I.

I6. W. H. Hudson, Un flâneur en Patagonie, trad. V. Llona, Paris, Stock, 1929 (désormais FP), p. II et W. H. Hudson, Idle Days in Patagonia, Londres, Chapman \& Hall, I893 (désormais IDP), p. I.

I7. R. Tomalin, W. H. Hudson. A Biography, ouvr. cité, p. 137. 
a été conduit en Patagonie par la "passion de l'ornithologie» (FP, p. I5; IDP, p. 5) et qu'il a été ravi de se trouver dans "ces déserts sauvages et lointains" $(F P$, p. I4; IDP, p. 5). Il veut y boire «à la coupe de la sauvage nature» ( $F P$, p. I7; $I D P$, p. 8$)$ qui est «à la fois si amère et si douce» ( $F P$, p. $22 ; I D P$, p. I3). Après quelque temps, il voyage pieds nus, ses bottes d'équitation étant devenues trop lourdes. Il s'enthousiasme à la vue des oiseaux de la famille des dendrocolaptidae, "petits architectes si industrieux" construisant des nids gigantesques, ce qui est «un labeur peu utile» (FP, p. I9; IDP, p. Io). En outre, Hudson est têtu, ou même un peu autodestructeur : souvent, il ne mange pas avec les autres, choisissant de "[se] consol[er] avec [sa] pipe, en [se] chauffant dans un silence pensif» (FP, p. 23; IDP, p. I4). Ainsi, la narration du premier chapitre se situe entre un roman d'aventures et un récit d'explorateur et d'ornithologue. C'est un récit linéaire de découvertes et d'observations qui est mené parallèlement à une progression linéaire vers une destination spécifique.

Pourtant, cette énergie qu'il déploie d'abord sans réserve pour aborder la Patagonie s'évanouit brusquement. Plus précisément, ces éclats d'énergie disparaissent au niveau factuel en prenant une forme différente au niveau fictionnel. Dans les faits, ses explorations doivent s'arrêter parce qu'il est grièvement blessé au genou et obligé de rester oisif. Sans préciser ce qui s'est passé auparavant, il raconte les circonstances de l'accident. Il faut noter que l'incident qui a provoqué son oisiveté forcée fut une expérience de mort imminente; il survit à une nuit sur le seuil de la mort. Alors que cela avait dû être très douloureux, il écrit plus tard à propos d'un grand nombre de pointes de flèches qu'il avait trouvées, puis perdues :

Le meilleur de mes trésors, les objets les plus curieux et les plus beaux que je pus choisir, emballés à part pour plus de sécurité, furent malheureusement perdus en cours de route - coup sévère dont je souffris davantage que de la blessure que j'avais reçue au genou. (FP, p. $47 ; I D P$, p. 38$)$

En ce qui concerne cette blessure, si douloureuse qu'elle ait pu être, elle lui permit d'adopter un nouveau rôle, celui de l'oisif. Les premiers paragraphes du second chapitre intitulé "How I became an Idler» ("Comment je devins un oisif»), constituent une digression achronologique à l'intérieur d'une digression, en relatant les pensées qui l'ont traversé après l'accident, avant même de décrire l'accident lui-même. Ensuite, le chapitre introduit, en justifiant également, au moins au début, les circonstances qui ont donné lieu à ce genre particulier de livre, c'est-à-dire l'époque pendant laquelle il est devenu un autre type d'aventurier, à savoir un aventurier de l'esprit. D'une manière comique, Hudson explique que «ces chapitres décousus, dont on pourrait dire qu'ils constituent le registre de tout ce que je n’ai pas fait, n’auraient jamais été écrits» (FP, p. 27 ; IDP, p. I8). 
En fait, la plupart des chapitres du livre sont plus compréhensibles lorsqu'on les lit en creux. L'auteur lui-même les traite avec une certaine négligence, quand il compare les problèmes dont il s'occupait à des mouches - probablement une des espèces les moins inspirantes de la terre - mais qu'il nomme malgré tout "ces insectes insaisissables et captivants de l'esprit» (FP, p. 28; IDP, p. 19). Cette tournure illustre la tendance d'Hudson à amalgamer des attributs humains et amorphes, ou même sa propre personne et des oiseaux. Ils sont transformés en "vagabonds ailés» et en "voyageurs» (FP, p. I5; IDP, p. 5). Dans les premières phases de son oisiveté forcée, son état d'esprit se caractérise par un mélange de résignation palpable et de ressassement mélancolique des mêmes idées, qui se lie à une narration délibérément de plus en plus non linéaire. Il constate : «Moi, je ne capturai ni ne découvris rien; pourtant, ces journées d'oisiveté forcée ne me furent pas pénibles.» (FP, p. 29; IDP, p. 20) Il écoute des histoires que des indigènes lui racontent mais en réalité ne s'y intéresse pas vraiment :

Je pouvais toujours les quitter sans regret pour m'étendre sur l'herbe, pour lever les yeux vers les arbres ou vers le ciel bleu et méditer sur toutes les choses imaginables. Mais, le jour où je n'eus plus la moindre excuse pour rester inactif, l'usage avait nourri en moi une habitude, celle de l'indolence. Elle est fort commune parmi les habitants de la Patagonie et semble convenir parfaitement à ce doux climat; cette habitude, cette humeur, je les conservai. Elles furent coupées de temps à autre par quelque recrudescence d'activité, pendant toute la durée de mon séjour.

Notre vie à l'état de veille ressemble parfois à un rêve. Le rêve se déroule avec assez de logique jusqu'à ce que le stimulant d'une sensation nouvelle, venue de l'intérieur ou de l'extérieur, le jette dans une confusion momentanée ou suspende son action; il reprend ensuite son cours, mais avec de nouveaux personnages, de nouvelles passions, de nouveaux motifs, un argument nouveau. (FP, p. 29-30; $I D P$, p. 20 ; je souligne)

Ce qui est frappant à première vue, en particulier en ce qui concerne le statut de l'oisiveté (ou de l'inactivité) dans le contexte victorien, est qu'Hudson, au moins au début de son texte, observe que les paysages de Patagonie lui permettent de transcender son état d'esprit coutumier qui toujours lui donne prétexte à être inactif. Le moment de dépassement d'une habitude désormais ancrée est central ici; il a été remarqué que la Patagonie est un espace liminaire qui a un effet particulièrement dynamique sur l'imagination humaine ${ }^{18}$. Les plaines de Patagonie semblent ne pas avoir de frontières et leurs images sont

toujours reliées tant à l'idée du monde [...] qu'à celle d'une infinitude chronotopique qui s'étend entre la modernité et le barbarisme. De ce point de vue, la

18. Voir aussi Ch. Moss, Patagonia: A Cultural History, Oxford, Oxford University Press, 2008. 
Patagonie est une zone paradoxale dans laquelle l'absence de frontières trouble une conception de la raison basée sur des limites et des échelles ${ }^{19}$.

Au lieu de l'appeler une «zone paradoxale» sans limites, on peut parler d'un espace liminaire qui trouble Hudson et en particulier sa manière d'exprimer ses pensées et ses perceptions. Ainsi, cet espace expérimenté d'une manière originale provoque un changement dans ses habitudes très enracinées, celle de l'indolence, celle de contempler le ciel en méditant. Tandis qu'à première vue, son comportement - son refus évident de fréquenter les indigènes - peut apparaître comme étant un peu arrogant, il ne doit pas être compris comme un rejet de toute interaction humaine, mais simplement comme un choix en faveur de la communion avec la nature. Par conséquent, lui-même transforme l'espace - qui se révèle habité par des êtres humains cherchant à communiquer - en un espace d'isolement. Cet isolement, qu'il s'inflige véritablement lui-même, lui permet de s'approprier l'espace étranger et de le transformer en un refuge facilitant le déploiement de son habitude d'indolence nouvellement acquise.

On peut soutenir que le topos d'une nature vierge en tant que locus amoenus, qui offre confort et sécurité, apparaît souvent, non seulement dans les textes de l'Antiquité classique et de la Renaissance, mais aussi, sous une forme moins sauvage, dans la poésie romantique et dans sa mise en valeur du motif pastoral, comme dans la poésie de William Blake $e^{20}$. Regarder la manière dont Hudson reprend ce topos classique et le modifie est intéressant. Jenny Haase constate qu'il ajoute une nouvelle dimension au topos de l'espace vide et des plaines stériles : son esprit a besoin de paysages désolés pour arriver à un état de paix intérieure et pour apprécier la nature dans toute sa beautéer. Cependant, selon moi, Hudson fait plus encore : tandis que normalement c'est le paysage ou le lieu naturel qui a un effet réconfortant sur l'individu, dans le cas d'Hudson, le rapport qui se met en place entre lui et la nature est bien davantage bilatéral. En d'autres termes : en s'isolant des indigènes, et en abordant la nature avec son état d'esprit oisif, il compose le paysage patagon de manière à faciliter ses réflexions. Le commentaire qu'il fait dans le passage cité ci-dessus précise les caractéristiques de son locus amoenus: en lien avec son attitude oisive, le lieu provoque des réflexions qui ne se réalisent ni dans un état onirique, ni dans un état d'éveil, et leur parcours est altéré par des influences internes ou

19. G. Nouzeilles, «Patagonia as Borderland: Nature, Culture, and the Idea of the State», Journal of Latin American Cultural Studies: Travesia, no 8, I, 1999, p. 35-48, p. 35.

20. Deux autres exemples sont la poésie de R. Burns qui était à la fois paysan et poète (rural), et The Solitary Reaper de Wordsworth.

2I. Voir J. Haase, Patagoniens verflochtene Erzählwelten. Der argentinische und chilenische Süden in Reiseliteratur und historischem Roman (1977-1999), Tübingen, Niemeyer, 2009, p. 77. 
externes; les repères habituels deviennent caducs. Cet état indéterminé peut aussi expliquer pourquoi, dans Un flâneur en Patagonie, on ne trouve pas une séparation stricte entre des thèmes intellectuellement exigeants et, d'autre part, des thèmes comiques et étranges. Ainsi, des observations scientifiquement fondées sur le lien entre le chant des oiseaux et la couleur de leur plumage sont juxtaposées à l'histoire pittoresque de son amitié avec un vieux chien. Le choix d'alterner entre deux types d'informations complètement différents est aussi une transgression. Hudson joue avec l'idée que son texte est une zone liminaire qui ne permettra jamais à ses lecteurs d'anticiper ce qui suit.

Dans le troisième chapitre, l'autocaractérisation d'Hudson et même son autocélébration en tant qu' "oisif" sont en plein essor : tout au long du chapitre, il décrit comment il passe ses journées à visiter à pied de nombreux villages de la zone, et comment il se promène le long de la rive en observant la faune et la flore et en faisant de nombreuses observations qui présentent un intérêt socioculturel. À titre d'exemple, dans certains villages, il trouve «des traces d'un système qui ressemblait à la division du travail, à une individualité chez l'ouvrier et à un goût artistique ou esthétique caractérisé» (FP, p. $48 ; I D P$, p. 39).

Aussi - et il faut le lire en lien avec l'admiration d'Hudson pour «l'esprit sauvage », un type d'esprit précivilisationnel et non corrompu - il trouve qu'en Patagonie «le contact avec une race supérieure [...] a avili [ce peuple] et a causé [sa] destruction" (FP, p. 49; IDP, p. 40). Il prédit que bientôt ils auront progressé vers «un état supérieur» et que leur esprit ne sera plus «sommei[llant] » (FP, p. 50; IDP, p. 40). Il pourrait bien s'agir ici d'expressions influencées par la terminologie darwinienne, sauf que pour Hudson, cet "état supérieur» n'est, en fait, pas désirable. Dans un autre chapitre intitulé "Concerning Eyes» ("En ce qui concerne les yeux»), Hudson détaille les nombreuses nuances et couleurs qu'il a observées dans les yeux des oiseaux. Il mentionne aussi les yeux des animaux nocturnes et relate comment il a tué un grand-duc de Magellan déjà blessé pour l'envoyer au musée. Il raconte combien il est resté longtemps hanté par ses yeux luisants de dragon. Il poursuit ses observations et pensées sur les yeux humains, et affirme comment, selon lui, "on ne peut s'empêcher de penser que les différentes races et tribus d'hommes, diverses quant à la couleur de la peau, [...] devraient posséder des yeux de couleurs variées" (FP, p. 197; IDP, p. 195). S’ensuivent de nombreuses rêveries dans lesquelles il décrit des iris d'or pâle ou parsemés, ou qui semblent même ornés de lettres de l'alphabet grec. Vers la fin du chapitre, Hudson décrit comment il se promène dans différents quartiers prospères ou pauvres de Londres pour découvrir la répartition de la couleur des yeux selon les différentes couches sociales. Dans ce but, il va et vient en regardant chaque passant directement dans les yeux (mais souvent en réussissant seulement à jeter un rapide coup 
d'œil) et en vient à conclure que l'œil britannique caractéristique de cette époque est d'une couleur grise impure.

En tenant compte du caractère quelque peu particulier d'Hudson et de ces raisonnements, qui pourraient être qualifiés de politiquement incorrects (mais qu'il a seulement menés parce qu'il aimait observer les gens), on peut présumer qu'il voulait exprimer son intérêt pour l'humanité tout entière et son admiration pour les habitants de Patagonie en particulier. Selon son point de vue, ils sont en communication avec la nature et ont des esprits «sommeillants» dans le sens positif du terme. Par conséquent, pour lui, «l'état d'esprit particulier » qu'il est fier d'arborer en Patagonie peut être attribué à "un retour à une condition mentale primitive et sauvage, à un état d'éveil et de vigilance intense, cependant dégagé des facultés mentales supérieures ${ }^{22} »$.

Il semble en fait que, lorsqu'Hudson parle de lui-même comme d'un paresseux qui, délicieusement "[c] ouché sur l'herbe» (FP, p. 42; IDP, p. 33) ou faisant des "promenades solitaires" et buvant à la " "douce et amère coupe de la Nature" " (FP, p. 44; IDP, p. 34), il veuille montrer qu'il peut être comme ces Patagoniens qu'il admire tant et dont il a intériorisé l'habitude d'indolence.

En outre, cette autocaractérisation est nécessaire pour qu'il puisse affirmer son rôle de voyageur. Il reprend des pratiques romantiques, les modifie et se trouve donc engagé dans un processus de réaffirmation du sujet. Vers la fin de son livre, Hudson élabore l'idée de cet état d'esprit particulier qu'il a pu cultiver en Patagonie. En effet, il parle avec insistance (et vers la fin, avec quelques redondances $\left.{ }^{23}\right)$ de son état de vigilance et de la manière dont il était capable d'agir, ou plutôt de penser inconsciemment (voir FP, p. 215-216; IDP, p. 2I4), lorsqu'il était entouré du silence patagon :

Toute la journée le silence me semblait délicieux; il était parfait et très profond. [...] Un jour que jécoutais le silence, je me demandai soudain l'effet que je produirais si je me mettais à crier. Sur le moment, cela me parut une horrible suggestion de l'imagination, une "pensée criminelle et incertaine», qui me fit presque frissonner, et je m'empressai de la chasser de mon esprit. Mais pendant ces journées de solitude, il était rare qu'une pensée quelconque passât dans mon esprit; des formes animales ne traversaient point mon champ visuel, des voix d'oiseaux n'assaillaient guère mes oreilles. Dans le nouvel état d'esprit où je me trouvais, la pensée était devenue impossible. Ailleurs, j'avais toujours pu penser librement à cheval; dans les pampas, aux endroits les plus solitaires, c'était quand je voyageais au grand

22. J. F. James, «Idle Days in Patagonia by W. H. Hudson. Review», Science, n 2I, 535, I893, p. $247-248$.

23. Vers la fin de son journal de voyage, sa manière de s'attarder sur l'idée d'un état d'éveil et de vigilance intense dans l'esprit sauvage et son appel à retourner à cet état deviennent un peu longs et répétitifs. Aussi résume-t-il cette partie sur un ton didactique telle une leçon qu’il a «apprise dans la solitude patagone» (FP, p. 233 et IDP, p. 230). 
galop que mon esprit s'enflammait. À présent, un cheval entre les jambes, j'étais devenu incapable de réflexion : mon esprit avait soudainement perdu sa nature de machine à penser : il s'était transformé en une machine destinée à je ne sais quelle fonction inconnue. Penser, c'était mettre en mouvement dans mon cerveau un appareil bruyant; or il y avait dans cette région quelque chose qui m'ordonnait de demeurer tranquille, et j'étais forcé d'obéir. J'étais en suspens et aux aguets; cependant je ne m'attendais jamais à rencontrer une aventure, et je me sentais aussi libre d'appréhension que je le suis aujourd'hui, assis dans une chambre, à Londres. Le changement qui s'était opéré en moi était aussi grand et aussi surprenant que si j'avais troqué mon identité contre celle d'un autre homme ou d'un animal; mais à l'époque, j'étais incapable de m'en étonner ou de faire des suppositions sur ce point; l'état me semblait familier plutôt qu'étrange, et bien qu'il s'accompagnât d'une forte sensation d'épanouissement mental, je ne le savais pas - je ne sus que quelque chose s'était passé en moi et mon intellect, que lorsque je l'eus perdu pour retourner à mon ancien moi, à la pensée et à la vieille existence insipide. (FP, p. 216-2I8; IDP, p. 215-216, italiques dans le texte original anglais)

Ce passage est central dans la mesure où il définit la nouvelle attitude d'Hudson envers la manière de penser influencée par l'environnement patagon, et sa conception très spécifique de l'oisiveté. Il décrit son cerveau comme une «machine à penser» qui est normalement mise en marche bruyamment lorsque le processus de pensée commence. Pourtant, entouré du silence "parfait» et "profond» de la Patagonie, sa «machine à penser» poursuit soudainement un objectif nouveau qui lui est inconnu. Au lieu de faciliter le procès mental (créatif) - trope commun que l'on trouve chez les écrivains de voyage - chez Hudson, le silence possède manifestement une qualité auditive : il peut l'écouter, et en effet, l'écoute absorbe toute son énergie de sorte qu'il est incapable de se servir de ses capacités intellectuelles pour se concentrer sur autre chose. C'est un type de silence qui met son esprit, quasi littéralement, dans un état oisif, un peu comme des machines ou moteurs qui ne fonctionnent pas, qui sont "oisifs", c'est-à-dire au point mort. Cet état le rend incapable de penser. En fait, pour Hudson, penser est un fardeau. Dans la dernière phrase de la partie citée, il y fait référence comme à un trait caractéristique de «la vieille existence insipide». Selon ce passage, la raison principale pour laquelle il a une opinion si négative de l'activité intellectuelle est qu'elle provoque généralement un état d'appréhension. Un état d'esprit oisif procure alors du réconfort. Le fait qu'il est manifestement capable de retrouver cette attitude lorsqu'il est "assis dans une chambre, à Londres" prouve, au moins implicitement, qu'il a été exposé assez longtemps au silence de Patagonie pour que le fait d'écrire à son propos permette de refroidir sa «machine à penser» lorsqu'il est dans un environnement différent. En mettant l'accent de façon si catégorique sur le fait d'avoir passé beaucoup de temps seul (et de l'avoir savouré), d'avoir marché sans même avoir utilisé d'autres moyens de transport, avant tout engagé dans 
une observation "profonde», il se distingue du touriste victorien arrogant et se présente comme un voyageur postromantique.

Il est frappant de constater que lorsqu'il se décrit lui-même, le mot "oisif» a, sans ambiguïté possible, un sens positif. C'est même quelque chose dont il se vante, tel un symbole de statut social. Pourtant, occasionnellement, il s'imagine être suivi d'une personne qui veut savoir comment travaille un naturaliste sur le terrain, et il est inquiet et honteux à l'idée que quelqu'un puisse le voir faire des choses inutiles comme par exemple brûler un arbuste en fleur (voir $F P$, p. I44; IDP, p. $\mathrm{I4}^{24}$ ). Il essaie de justifier son comportement par le désir humain d'échapper aux "chaînes de la convention » et d'agir comme «des singes en liberté, sans entrave à [leurs] gambades» ( $F P$, p. I45; IDP, p. I43); dans cet extrait, le côté négatif de l'oisif malicieux et destructeur prévaut. Aussi, lorsqu'il emploie cet adjectif pour décrire le sens d'une question, celui-ci prend une connotation clairement négative. Par exemple, lorsqu'il médite sur les crânes qu'il a trouvés dans une vallée, il constate que partout ailleurs qu'en Patagonie se demander quelle image du monde on aurait si l'on était capable de voir à l'intérieur de ces cavités vides, serait "simplement idle (vain) », c'est-à-dire futile ou étrange (voir FP, p. 5I ; IDP, p. 44).

Lorsqu'un ornithologue a honte parce qu'il est conscient de ne pas être un naturaliste modèle mais simplement un oisif qui écrit sur ses divagations et qui ne représente donc pas convenablement la profession, devrait-on alors penser, comme le fait Pound, qu'il est un poète (et non pas un naturaliste) qui s'est égaré dans la science? La plupart des critiques du Flâneur jugent que le journal de voyage a une qualité plus poétique que scientifique. Ainsi Brickell parle des "pages d'une beauté inoubliable sur le chant des oiseaux ${ }^{25}$ ». Une critique dans la revue Saturday Review relève aussi ce passage et en fait l'éloge comme révélant "un sens esthétique remarquablement $\operatorname{aigu}^{26}$ ». Il y a en effet encore beaucoup d'autres passages qu'on serait tenté de qualifier de magnifiques. Selon moi, leur beauté provient en partie de leur originalité : il

24. Nietzsche résume ce dilemme d'une manière fort poignante dans le chapitre "Muße und Müßiggang" ("Loisir et oisiveté») de son livre Die fröhliche Wissenschaft (Le Gai Savoir) (I882) où il fait le lien, d'une part, entre repos et oisiveté (otium) et, d'autre part, entre honte et mauvaise conscience. Lorsqu'il s'interroge sur l'enthousiasme avec lequel beaucoup d'Américains creusent le sol pour trouver de l'or, un enthousiasme que l'on imite aujourd'hui dans la "Vieille Europe», il écrit : "On a déjà honte, aujourd'hui, du repos; la méditation prolongée provoque presque des remords. On pense la montre en main, comme on déjeune, le regard rivé au bulletin de la Bourse, - on vit comme un homme qui constamment "pourrait rater" quelque chose.» (F. Nietzsche, Le Gai Savoir, trad. P. Wotling, Paris, GF Flammarion, 2007, p. 264.)

25. H. Brickell, «W. H. Hudson», art. cité, p. 99.

26. 1893 , p. 36r. 
n'utilise que rarement des lieux communs pour décrire la nature et, comme le remarque un critique, il n'est «jamais terne, maladroit ou lourd ${ }^{27}$ ». C'est la contribution d'Hudson que de vivifier "de signification ce qui est trivial ou général ${ }^{28}$ " et de soumettre chaque observation au filtre de son imagination, ce qui réduit le risque de répéter des lieux communs lorsqu’il décrit des phénomènes naturels. Il souligne explicitement sa conscience de soi grandissante en tant qu'observateur d'objets, et en particulier de la nature, lorsqu'il parle de sa vigilance. Tout comme dans la description des couleurs des yeux ou dans le dialogue qu'il envisage avec les crânes de la vallée du cimetière indien, les choix qu'il fait au niveau du contenu, c'est-à-dire "le chemin pittoresque qu'empruntent ses réflexions ${ }^{29}$ ", de même que le style qu'il adopte pour les peindre sont atypiques et donc surprenants.

Le terme que ces critiques évoquent chez le lecteur est celui de la «littérarité", concept introduit par le formaliste russe Roman Jakobson. La question de savoir si le texte d'Hudson est littéraire est pertinente dans la mesure où elle s'insère dans le débat sur l'existence de traits caractéristiques du genre $\mathrm{du}$ journal de voyage. Pour examiner la pertinence de ce terme pour le texte d'Hudson, je me propose d'examiner en détail un passage en particulier. Dans celui-ci, Hudson décrit une promenade à cheval particulièrement rapide et les impressions qu'il a recueillies ce jour-là.

À ses yeux, les couleurs qu'il voit agissent comme un stupéfiant :

Mais au bout d'un certain temps le soleil, s'inclinant vers l'ouest, commença à briller derrière nous par des déchirures dans les nuages, et devant nous, sur les nuages qui s'enfuyaient à toute vitesse, apparut un arc-en-ciel aux teintes si vives que nous poussâmes des cris de joie. Pendant près d'une heure, nous chevauchâmes avec cette vision de gloire devant les yeux; dans notre course rapide, nous passions successivement devant des bosquets de saules défeuillés et noirs d'écorce, sur notre droite, et sur la gauche, devant des collines couvertes de ronces et toutes grises, tandis que de grandes bandes d'oies se levaient devant nous en poussant des sifflements aigus entremêlés de cris solennels et ronflants; le grand arc de feu et de nuées vivait encore, s'évanouissant par moments quand s'amincissaient les lambeaux de nuages, puis, quand on croyait qu'il allait disparaitre, il se ravivait avec une nouvelle et plus merveilleuse splendeur [...].

Je ne suppose pas que les couleurs étaient en réalité plus vives que celles des nombreux arcs-en-ciel que j'ai pu voir ailleurs; c'était, je crois, le gris universel de la terre et du ciel, dans cette grise saison d'hiver et dans une région où la nature emploie si économiquement la couleur, qui donnait à celui-ci une beauté si sublime qu'elle produisait sur nous l'effet du vin.

27. E. J. Roberts, «W. H. Hudson, Naturalist and Author», The Baptist Quarterly, n 8, 4, 1936, p. I9I-I99, p. I9I.

28. Saturday Review, 1893, p. 36r.

29. Ibid. 
"Les yeux», a dit Bacon, "ne sont jamais aussi flattés que lorsqu'ils contemplent une gaie broderie sur un fond triste et sombre». L'arc vert et vide tendu sur une vapeur gris d'ardoise nous enseigna cette vérité. Mais la nature est trop sage "Pour émousser d'un plaisir rare / La fine pointe». Le jour de la splendeur et de la gloire surnaturelles ne vient qu'après bien des jours qui ne sont que naturels et neutres de couleurs. On l'attend, on le guette, et quand il arrive, il est comme un grand jour de fête et de réjouissance, le jour où la paix a été conclue, où notre amour nous a été rendu, où un enfant est né. De tels spectacles sont comme certains sons, qui non seulement nous ravissent par leur pure et belle qualité, mais éveillent en nous des sensations que nous ne saurions ni analyser ni approfondir. Ils nous sont familiers, et plus étranges pourtant que les choses les plus étranges, avec une beauté qui n'est point de la terre, comme si un ami bien aimé, mort depuis longtemps, nous regardait du haut du ciel, transfiguré. Il me semble étrange que, à notre connaissance du moins, les Incas aient été les seuls adorateurs de l'arc-enciel. (FP, p. 62-63; IDP, p. 53-55; je souligne.)

Ce qui est remarquable, c'est que même si ce passage esquisse des scènes de joie irrationnelle et exubérante, et si quelques-unes des expressions sont manifestement difficiles à interpréter (particulièrement «la journée de splendeur et de gloire»), Hudson, ou plutôt sa voix narrative, garde le contrôle des événements. Il est bien conscient que l'arc-en-ciel découvert ce jour-là, qui est l'élément déclencheur de l'éclat poétique, n'était en rien différent de ceux vus dans le passé. D'autre part, il prive consciemment la scène de son mystère et de son illogisme peut-être magnifique, ce qui ne peut pourtant pas être une manœuvre stratégique pour un écrivain voulant envoûter ses lecteurs. Mais à un niveau littéraire et textuel, il réussit à composer un texte qui peut avoir un effet envoûtant précisément parce qu'il est finement élaboré et construit à partir de plusieurs moyens stylistiques. Il ne décrit pas seulement les moments qui précèdent la rencontre finale avec l'arc-en-ciel majestueux d'une manière très stylisée, en utilisant des répétitions, soit "grove after grove» ("bosquets»), " hill after hill» ("collines»), "thinner and thinner» ("s'amincissaient») et une onomatopée, "shrill whistlings» ("sifflements aigus»), mais l'auteur se construit aussi sa propre approche d'observation. Même s'il a manifestement été bouleversé par le phénomène naturel dont il a été témoin ce jour-là, il réussit à introduire une voix narrative rationnelle et même avisée. En citant Bacon et un sonnet de Shakespeare ("To blunt the fine point of seldom pleasure» provient du sonnet 52), il trouve une explication logique pour l'image vive qui s'est gravée dans son esprit, grâce à l'interaction du soleil et des nuages : cette image se détache du fond terne et dans l'ensemble gris du paysage hivernal environnant. Ainsi enivré, il a, en un sens, un prétexte pour dériver vers ce style imagé et pour comparer ses impressions visuelles, de manière synesthésique, à des sons. Il en conclut que les humains ne sont pas capables de répondre différemment 
à de telles situations parce qu'elles se situent au-delà du domaine de l'analyse, comprenons qu'elles ne peuvent pas être mises en mots.

C'est pourquoi la conclusion d'Ezra Pound est pertinente, selon laquelle «Hudson est un excellent exemple de la théorie de Coleridge du "miracle qui peut être accompli simplement par le fait que quelqu'un puisse ressentir une chose plus intensément ou la connaître plus intimement qu'elle ne l'a été jusque-lă" ${ }^{\circ} »$. Les expressions oxymoriques de Pound sont à cet égard particulièrement appropriées. En effet, les moments d'oisiveté d'Hudson se caractérisent, tout comme l'espace patagon réapproprié où il se trouve, par leur nature liminaire et par le fait qu'ils sont tenus ensemble simultanément par la sensibilité et la pensée. Lorsqu'il compare l'étrange beauté du spectacle de l'arcen-ciel au regard inattendu d'un ami mort depuis longtemps, Hudson fait de la mort un exemple paradigmatique pour un concept qui surpasse l'imagination humaine. Il finit ainsi par révéler le lien paradoxalement proche - et même la similarité - entre les moments de beauté et de vivacité et l'annihilation de ceux-ci dans la mort. Par le degré d'élaboration de ce passage exemplaire, les critères de "littérarité» se réalisent : ainsi, l'hypothèse des Formalistes selon laquelle la «littérarité» distingue les textes littéraires des textes non littéraires (comme les journaux de voyage) se révèle arbitraire.

Selon David Miller, le passage présente des «moments épiphaniques qui donnent un aperçu du paradis, saisi grâce aux particularités d'une vie personnelle d'une manière aussi sûre que ne le sont les détails de la nature; et la révélation perdure comme quelque chose d'insaisissable ${ }^{3 \mathrm{I}} »$. La remarque de David Miller peut être appliquée à la fiction d'Hudson, qui est l'élément principal de son analyse. Dans Un flâneur en Patagonie, cependant, la notion d'un ou $d u$ paradis n'est pas centrale. L'usage du terme d'épiphanie par David Miller est néanmoins utile ici aussi parce qu'il admet l'énergie endothermique avec laquelle les observations d'Hudson sont menées. Toutes les phases intenses de contemplation oisive commencent avec des accès d'oisiveté qui l'amènent à «se promener continûment pendant des heures", faisant des pauses de temps en temps, par exemple pour écouter le chant d'un oiseau particulier ou pour s'allonger près d'une volée d'oiseaux jusqu'à ce que les oiseaux oublient sa présence (voir FP, p. I47; IDP, p. I45-I46). Ce n'est donc pas seulement le paysage patagon qui encourage l'oisiveté d'Hudson, mais c'est lorsqu'il y est propulsé par un éclat bref d'énergie, que sa déambulation dans ce nouvel espace commence. David Miller explique que «le moment épiphanique et

30. E. Pound, «Hudson», art. cité, p. I3.

31. D. Miller, "Introduction», dans id., W. H. Hudson and the Elusive Paradise, Londres, Macmillan, I990, p. I73-175, p. I74-175. 
archétypal dans l'œuvre d'Hudson perdure à rebours de la certitude rationnelle en tant que quelque chose de fracturé ou de résiduel, et pourtant étrangement insistant ${ }^{32} »$. Hudson célèbre une façon d'écrire qui se dresse à l'encontre des pratiques littéraires contemporaines plus communes telles que le réalisme, mais il reprend une tradition qui perdure dans l'armature conceptuelle. Cette mentalité se reflète dans son oisiveté et ses soudains accès d'écriture.

Dans un autre chapitre, Hudson médite pendant une dizaine de pages sur Moby Dick d'Herman Melville et particulièrement sur sa description de la blancheur de la baleine. Herbert Cahoon relève que bien que la dispute intérieure d'Hudson avec Melville soit longue, Melville n'est jamais mentionné par la critique en lien avec les travaux d'Hudson ou inversement, à part dans un bref commentaire de Morley Roberts ${ }^{33}$. Selon Herbert Cahoon, «le chapitre d'Hudson [...] est une œuvre solide écrite dans une prose musicale qui approche la perfection - ces qualités sont rarement absentes dans les livres du naturaliste ${ }^{34} »$. Le déclencheur immédiat en est les premières neiges qui, après quelques mois passés en Patagonie, rappellent à Hudson son arrivée au moment où il avait vu «la blancheur d'une mer tumultueuse et couleur de lait» et s'était trouvé en présence d' "une blancheur plus vaste et plus étrange encore» (FP, p. II3-II4; IDP, p. III-II2). Il juge ce plaisir purement intellectuel et rejette l'idée qu'il y ait chez l'être humain une pulsion instinctive qui le rende joyeux à la vue de la neige. Cela l'amène à examiner en détail le chapitre du roman de Melville, dans lequel la couleur blanche provoque, chez les hommes, une panique inexplicable et une angoisse instinctive. Mais Hudson le réinterprète comme un cas d'animisme, qui signifie pour lui que l'esprit se projette dans la nature. Ce qui amène cette méditation est un accès d'oisiveté et le contact direct avec la nature. Le chapitre entier qui n'a pas de lien direct avec le projet ornithologique, parait constituer un autre éclat poétique et un espace textuel d'oisiveté. Hudson en arrive à la révélation suivante :

Rappelons-nous que nos poètes ne parlent pas scientifiquement mais dans le langage de la passion, quand ils disent que le soleil se réjouit dans le firmament et se rit de la tempête; que la terre s'habille de fleurs au printemps et que les champs sont heureux en automne; que les nuages froncent le sourcil et pleurent, que le vent soupire et "profere des mots lugubres en voyageant». Ce ne sont pas là de simples métaphores comme on nous a appris à le dire. Dans les moments d'émotion, quand nous revenons aux conditions primitives de l'esprit, la terre et toute la nature sont vivantes et intelligentes, et elles sentent comme nous sentons.

\footnotetext{
32. Ibid., p. 2I.

33. M. Roberts, W. H. Hudson: A Portrait, Londres, Eveleigh Nash \& Grayson, I924, p. I43-I44.

34. H. Cahoon, "Herman Melville and W. H. Hudson", American Notes and Queries, n 8, 9, I949, p. I3I-I32.
} 
Quand, après une période de temps triste, le soleil brille d'un éclat inattendu, chaud et brillant, qui na pas senti dans ce premier moment de joie que toute la nature partage sa joie consciente? Ou, dans les premières heures d'une grande douleur, qui n’a pas éprouvé une sensation d'étonnement, et même, de ressentiment à la vue du ciel bleu et souriant et de la terre inondée de rayons de soleil ? [...] Il est sans doute vrai que nous ne sommes animistes à ce point qu'à de rares moments et dans des circonstances exceptionnelles et tant que durent certains aspects de la nature qui ne se présentent qu'à de rares intervalles. ( $F P$, p. I2I-I22; IDP, p. I2O-I2I)

Alors que la couleur blanche renforce la beauté des objets, selon Hudson, seulement pour certains d'entre eux, elle provoque aussi la peur : il s'agit là d'une distinction que l'on ne trouve pas chez Melville. Aussi discute-t-il en détail deux erreurs majeures que, selon lui, Melville commet. Même si certains objets blancs provoquent véritablement un sentiment de peur, cela n'est généralement remarqué que lorsque quelqu'un d'autre le souligne. La seconde erreur commise par Melville, selon Hudson, est qu'il appelle l'effet de la blancheur sur l'esprit une "expérience héritée» (FP, p. II9; IDP, p. II8) extranaturelle ou surnaturelle. Melville pense que c'est une erreur d'attribuer la cause de ce déclenchement de peur ou de stupeur à la blancheur d'un objet et non pas à l'objet lui-même. Après tout, les moutons et les agneaux sont également d'une blancheur pure, mais ne sont en règle générale pas craints par les êtres humains. Ainsi, il met sur le même plan la couleur blanche et toutes les autres couleurs vives, telles que l'or ou le rouge vif, et illustre cela avec l'exemple d'un guerrier qui combat une armée d'hommes vêtus de couleurs ternes : qu'il porte du blanc, de l'or ou n'importe quelle autre couleur vive, il attirera de toute façon les regards et provoquera la stupeur de tous. De surcroît, que la neige qui tombe en hiver mette fin à la saison des récoltes fait tout de même partie du savoir traditionnel; la neige est donc invariablement associée au froid et à la mort.

Cependant, Hudson ne s'arrête pas à un rejet de la position de Melville. Il présente sa propre explication pour cette sensation de stupeur et offre ainsi une solution qui répond aux erreurs commises par Melville : «c'est que ce quelque chose de mystérieux qui nous émeut à la vue de la neige provient de l'animisme qui existe en nous et de notre manière animiste d'envisager tous les phénomènes exceptionnels" (FP, p. I2O; IDP, p. II9). L'animisme peut être déclenché par toute sorte d'autres événements exceptionnels qui ont lieu dans la nature :

[il] signifie ici, non pas une doctrine d'âmes qui survivent aux corps et aux objets qu'elles habitent, mais la projection que l'esprit effectue de lui-même dans la nature, l'attribution qu'il fait à toutes les choses de sa propre vie sensitive et de son intelligence, cette faculté primitive et universelle sur quoi se fonde la philosophie animiste du sauvage. Quand nos philosophes nous disent que cette faculté 
est morte en nous, qu'elle a été efficacement tuée par le raisonnement ou qu'elle ne survit que temporairement chez nos enfants, je crois qu'ils se trompent, chose qu'ils pourraient découvrir par eux-mêmes si, laissant leurs livres et leurs théories, ils voulaient bien se promener la nuit dans le "Bois de Westermain", ou dans n’importe quel autre bois, puisque tous les bois sont enchantés (FP, p. I2I ; IDP, p. II9, je souligne).

Au lieu d'évoquer la projection de l'esprit sur la nature et ainsi de risquer de confondre l'animisme avec l'anthropomorphisme, on peut parler de la traduction de l'esprit dans la nature «jusqu'à ce que la scène visuelle participe à sa vie mentale et à son intelligence», un "processus mental » qui ne peut être «connu que par l'Homme doté d'imagination » alors qu' "une personne sans imagination n'expérimenterait que l'angoisse ${ }^{35} »$. Tout le monde n'est pas capable de pratiquer l'animisme et d'en jouir à la fois. C'est une capacité réservée à une minorité. Même si Hudson ne s'en vante pas, il se présente lui-même comme étant doué d'une sensibilité visuelle très raffinée. De plus, il revendique d'une manière à la fois nonchalante, agacée et plutôt énigmatique, être le détenteur d'un savoir "primitif universel » que les philosophes se sont employés à barrer pour eux-mêmes et pour les autres.

Le chapitre sur la blancheur de la baleine chez Melville n'est pas un compte rendu des pensées d'Hudson lors d'une journée particulière en Patagonie - elles ne sont donc spécifiques ni au temps ni au lieu. Il est plutôt issu d'une prise de notes du même jour ou de quelques jours plus tard qu'il a ensuite ordonnée lorsqu'il s'est souvenu de ce moment exceptionnel vécu lors des premières neiges. C'est une digression prolongée dans laquelle le temps des horloges ne joue aucun rôle, provoquée par une révélation qui prit forme lorsqu'il se trouvait au cour de la Patagonie sans que cette révélation soit directement reliée au paysage. Il s'agit d'un passage qui transforme l'oisiveté en un texte, qui réussit à extraire une forme "d'oisiveté portable» d'un moment exceptionnel.

Nombreux sont les passages qui, à l'instar du précédent prennent de plus en plus souvent, et parfois d'une manière irritante, la forme de ralentisseurs textuels. Un fait particulièrement intéressant pour mon analyse est qu'il s'agit majoritairement de passages qui décrivent des émotions ou des pensées liées à l'oisiveté ou qui sont même des moments d'oisiveté. Le narrateur se souvient d'avoir vu quelque chose qui l'a arrêté et plongé dans une contemplation. Celle-ci, à son tour, l'amène à réévaluer des notions qu'il a précédemment examinées. Alors que la rupture de style entre le premier et le second chapitre peut traduire une inconséquence au niveau du contenu ainsi qu'on l'a dit, elle est en fait pertinente sur le plan littéraire - l'oisiveté factuelle se reflète

35. Saturday Review, I893, p. 36I. 
textuellement dans des digressions qui sont davantage mises en relief parce qu'elles sont précédées par le chapitre introductif motivé par les événements extérieurs. Le second chapitre marque déjà une pause, il piétine, le temps du récit s'arrête ou bien celui du discours s'allonge considérablement (ou peutêtre le temps ne joue-t-il plus aucun rôle, puisqu'Hudson ne dit pas pendant combien de temps il a été contraint de garder le lit). Les deux passages analysés ci-dessus fonctionnent de la même manière.

Le dénominateur commun de tous ces passages lus comme des moments d'oisiveté est leur élaboration dans la solitude et dans un état d'esprit particulier favorisé par les paysages patagons. J'ai montré qu'Hudson savoure les moments où sa "machine à penser " est oisive et qu'il évite la compagnie et le bavardage des indigènes. La seule exception est son hôte, qui est pour lui un compagnon acceptable, et peut-être même une espèce de "guide» dans l'apprentissage de l'oisiveté, parce que «lui aussi est un flâneur, mais plus accompli [que lui-même] ne p[ourrait] jamais espérer de l'être» (FP, p. I37; IDP, p. I35). En général cependant, le fait d'être interrompu dans ses méditations solitaires est pour lui gênant et même douloureux. Lorsqu'il rêve par exemple, il rêve souvent de la découverte d'une nouvelle espèce d'oiseau, ce qui lui apporte la plus grande joie. Le réveil en devient un chagrin douloureux (voir $F P$, p. I5; IDP, p. 6). Il cherche le "vieux monde" et la solitude "pour connaitre une exquise sensation de soulagement, d'évasion et d'absolue liberté» (FP, p. I7; IDP, p. 8).

En I884, l'ami d'Hudson Morley Roberts lui présente l'écrivain Georg Gissing avec lequel il partage non seulement «l'expérience directe de la pauvreté et de l'écriture» mais aussi «l'habitude des promenades solitaires ${ }^{36}$ ». Ils échangent beaucoup de lettres et dans l'une d'elles, datée du 19 janvier I898, se référant à l'anxiété de Gissing, Hudson écrit :

Depuis longtemps je m'efforce de m'approprier une nouvelle philosophie de vie qui me sera d'une grande aide. Ce n'est pas que rien ne m'importe mais plutôt que c'est inutile de se battre contre les atteintes de la vie. Je trouve aussi des bénéfices à me couper de la vie et de son tumulte - à considérer avec distance ses événements sombres et lumineux, même lorsqu'ils me concernent directement, et à les voir comme de simples couleurs et motifs d'un tapis sur lequel je vais et je viens. Je pense qu'on peut faire un bon bout de chemin dans cette direction sans pour autant devenir un cynique. Le cynisme, j'imagine, est inné comme d'autres bonnes ou mauvaises choses - le tempérament artistique, la férocité, la fourberie, la bienveillance, etc. ${ }^{37}$

Hudson se désengage délibérément; cela ne veut pas dire qu’il vit comme un exclu de la société, mais qu'il s'est désengagé au quotidien à un niveau

36. R. Tomalin, W. H. Hudson, ouvr. cité, p. I2I.

37. D. Shrubsall et P. Coustillas (dir.), Landscapes and Literati, ouvr. cité, p. 32 (je souligne). 
microcosmique. Pour lui, c'est à la fois une philosophie de vie et, comme on peut le constater, une sensibilité individuelle. Avec celle-ci, il peut considérer avec distance les événements, tant négatifs (ce qui est le cas normal) que positifs (ce qui est quelque peu inhabituel), et les rendre abstraits à un tel degré qu'ils en deviennent de simples couleurs et motifs. S'il n'était pas doué de la faculté de contemplation, une telle stratégie mènerait certainement à l'anxiété et à la détresse. Mais ayant trouvé du réconfort dans cette approche de la vie, il suggère à son ami Gissing de faire la même chose.

Pendant ses recherches pour son livre, et pas seulement pendant son année en Patagonie, Hudson s'est absenté plusieurs fois et dans l'une de ses lettres à Gissing du 28 décembre 1896 , il fait référence au fait qu'il y a des jours où il n'est pas au courant de ce qui se passe dans le monde littéraire et scientifique et qu'il «devient de plus en plus un ermite dans sa cellule ${ }^{38}$ ». Que la solitude fût son mode de vie préféré, et non pas le luxe d'une dose occasionnelle d'évasion, cela devient évident lorsque vers la fin du Flâneur en Patagonie, il parle de Thoreau et de son erreur qui consistait à penser

que la société des êtres humains pourrait être essentielle à son bonheur; mais il sentait que cette humeur de son esprit était quelque peu insensée, il ne tarda guère à redevenir sensible à la douce et bienfaisante société de la nature, à une infinie et inexplicable amitié semblable à une atmosphère qui l'aurait soutenu $(F P$, p. 23I ; $I D P$, p. 228 ; je souligne).

Pour Hudson, chaque être humain à l'esprit raisonnable doit savoir que le bonheur et la douceur des mœurs peuvent seulement être ressentis dans une société particulière - non pas celle des humains, mais celle de la nature. Dans l'un des derniers chapitres, "Les plaines de la Patagonie», il affirme même qu'aller vers les plaines stériles et grises où il peut être seul est pour lui comme assister à «une fête» $(F P$, p. 213; IDP, p. 2II).

À titre de caractéristique plus générale de l'oisiveté chez Hudson, les ralentisseurs de son texte présentent des idées et des réflexions d'une nature si abstraite et générale que, même si celles-ci proviennent de Patagonie, elles se réferrent toujours à une "image globale». Ce qu'il dit, mis à part les observations ornithologiques (qui ne sont cependant jamais exprimées dans un style que l'on trouverait dans une encyclopédie d'histoire naturelle), n'est jamais spécifique au point de ne plus être significatif pour des lecteurs qui viendraient d'un pays ou d'un milieu social différent. Ses réflexions servent de point de départ pour des considérations supplémentaires. Ainsi, lorsqu'on considère ses remarques sur la solitude et le silence, et sur le type d'oisiveté qu'ils entraînent, la motivation

38. Cité dans ibid., p. 27. 
sous-jacente d'Hudson semble être la quête de la paix - non pas dans un sens politique, mais celle du microcosme individuel. Longtemps après son retour en Angleterre ${ }^{39}$, il encourage d'autres personnes à chercher une paix comparable à celle qu'il a connue en Patagonie et, ce qui est peut-être encore plus important, il fait l'éloge des aspects positifs de l'inactivité liée à la contemplation. Dans une lettre à Gissing du 7 avril I897, il exprime sa surprise d'entendre que celui-ci n'a pas réussi à trouver la paix à Devon où il séjourne à ce moment-là et où il passe beaucoup de temps dehors. Il lui demande :

Si ce n'est pas dans une contrée verte - je suis souvent resté béat, dans une sorte de stupeur d'émerveillement face au vert quasi surnaturel - où la paix peut-elle siéger? J'ai longtemps choyé la faculté ou même l'art béni de l'oubli, celle d'oublier complètement ce qu'il a été douloureux ou vain de se rappeler; et j'ai développé l'idée selon laquelle dans cette région de "South Devon» un tel état d'esprit peut être plus facilement atteint que dans la plupart des autres lieux ${ }^{40}$.

La Patagonie et le "South Devon» deviennent remarquablement similaires parce que, dans les deux cas, Hudson réduit ces régions à des paysages sonores de bruit blanc : ils lui permettent d'éteindre sa "machine à penser", d'être captivé dans une "sorte de stupeur d'émerveillement» et de voir la "paix». Plusieurs décennies après avoir vécu pour la première fois l'expérience de l'écoute du silence, il peut alors écrire à Gissing et l'encourager à l'imiter.

Lorsque nous essayons de résumer la conception de l'oisiveté d'Hudson selon ce qu'il en dit dans Un flâneur en Patagonie, nous nous trouvons face à un défi parce que nous sommes distraits par le bruit de notre propre «machine à penser ». Hudson suggère qu'il a connu des moments pendant lesquels ce bruit a été éliminé de sa vie. Il essaie de décrire ce qu'un esprit oisif pourrait potentiellement ressentir - ce projet devait rester à l'état d'expérimentation parce qu'il est une tentative d'écrire au sujet de ce qui arrive lorsqu'on a oublié qu'on est doué de la faculté d'écrire. Tous les chapitres considérés conjointement se composent d'éclats équilibrés écrits par un naturaliste de terrain autoproclamé. Comme il l'a dit dans une conversation avec Ford Madox Ford

39. Il n'était pas exclusivement un ermite. Après le succès de ses romans, il devint assez célèbre, particulièrement parmi les autres écrivains de son époque. Il prenait part aux «déjeuners du mardi » du restaurant français Mont Blanc, situé dans la Gerrard Street dans le quartier de Soho de Londres "où [...] la haute société intellectuelle de la ville déjeunait et discutait avec une sobriété grave les problèmes sociaux de l'époque [...]. D'habitude il y avait Conrad et Galsworthy et W. B. Yeats et Mr. Hilaire Belloc et Muirhead Bone, le graveur, et Sturge Moore, le poète [...]. Et puis soudain, dans un silence, on entendait le bruissement des branches vertes de la New Forest de Hampshire ", c'est-à-dire l'arrivée d'Hudson (F. Madox Ford, «W. H. Hudson », art. cité, p. 306).

40. Cité dans D. Shrubsall et P. Coustillas (dir.), Landscapes and Literati, ouvr. cité, p. 28. 
qu'il appelait un «styliste»: "Je ne suis pas un artiste. C'est le dernier terme que j'utiliserais pour me désigner. Je suis un naturaliste de terrain qui écrit ce qu'il voit... C'est parfaitement simple d'écrire ce qu'on a vu. Tu pourrais le faire si tu le voulais. Les yeux fermés ${ }^{41} »$. D'une certaine façon en effet, l'idée sous-jacente à l'oisiveté d'Hudson est simple : elle peut être atteinte lorsqu'on se met à voir, les yeux fermés, et qu'on n'est pas dérangé par le bruit de ses propres pensées.

4I. F. Madox Ford, «W. H. Hudson», art. cité, p. 314. 